\title{
Probing a degenerate-scalar scenario in a pseudoscalar dark-matter model
}

\author{
Sachiho Abe, ${ }^{1}$ Gi-Chol Cho $\odot,{ }^{2}$ and Kentarou Mawatari $\odot^{3}$ \\ ${ }^{1}$ Graduate school of Humanities and Sciences, Ochanomizu University, Tokyo 112-8610, Japan \\ ${ }^{2}$ Department of Physics, Ochanomizu University, Tokyo 112-8610, Japan \\ ${ }^{3}$ Faculty of Education, Iwate University, Morioka, Iwate 020-8550, Japan
}

(Received 20 January 2021; accepted 5 August 2021; published 25 August 2021)

\begin{abstract}
We study a pseudoscalar dark-matter model arising from a complex singlet extension of the standard model (SM), and show that the dark-matter nucleon scattering is suppressed when two $C P$-even scalars are degenerate. In such a degenerate-scalar scenario we explore the model parameter space which satisfies constraints from the direct detection experiments and the relic density of dark matter. In addition, we discuss a possibility to verify such a scenario by using the recoil-mass technique at the International Linear Collider. We find that a pair of states separated by $0.2 \mathrm{GeV}$ can be distinguished from the single SM-like Higgs state at $5 \sigma$ with an integrated luminosity of $2 \mathrm{ab}^{-1}$.
\end{abstract}

DOI: 10.1103/PhysRevD.104.035023

\section{INTRODUCTION}

The existence of dark matter (DM) has been suggested by several cosmological observations, e.g., precise measurements of the cosmic microwave background [1]. Although weakly interacting massive particles (WIMPs) are known as a good candidate of DM, no signature of the WIMP DM has been found yet in particle physics experiments. Among various WIMP-DM searches, direct detection experiments such as XENON1T [2] have significantly improved the upper bounds on the cross section between DM and nucleons, so that some new physics models which predict the DM candidate are severely constrained.

An interpretation of the null result in the direct DM detection experiments is that the DM is heavy $[\gtrsim O(\mathrm{TeV})]$ or light $[\lesssim O(\mathrm{GeV})]$ enough. Another possibility to explain the result is that effective interactions between DM and nucleons are suppressed for some reason. For example, it has been known that the tree-level amplitudes of DM-quark scattering in the nonrelativistic limit are proportional to the momentum transfer in pseudoscalar portal models $[3,4]$ so that the amplitudes are suppressed at the low-energy limit. The estimation of the higher-order contributions in such models and testability of those effects at future direct detection experiments are given in Ref. [5].

Another example of suppression of the DM-quark scatterings at the low-energy limit has also been shown in Ref. [6], where the DM is a pseudo-Nambu-Goldstone

Published by the American Physical Society under the terms of the Creative Commons Attribution 4.0 International license. Further distribution of this work must maintain attribution to the author(s) and the published article's title, journal citation, and DOI. Funded by SCOAP ${ }^{3}$. boson as a consequence of a global U(1) symmetry breaking of the scalar potential. Such a model is known as the minimal pseudo-Nambu-Goldstone DM model arising from a complex singlet extension of the standard model (CxSM) [7] with a softly broken U(1) symmetry by the DM mass term. In this model, the DM-quark scatterings are mediated by two scalar particles. The amplitudes of the DM-quark scattering processes mediated by each scalar particle have the same magnitude at zero momentum transfer but the opposite relative sign, and hence the scattering between the DM and nucleons is highly suppressed at low energy. The model has been studied in various contexts, e.g., in Refs. [8-15]. It is known, however, that the minimal model suffers from the so-called domain-wall problem because the $Z_{2}$ symmetry of the scalar potential is spontaneously broken when the singlet field develops the vacuum expectation value (VEV) [16].

In this paper, we adopt the most general renormalizable scalar potential of CxSM with soft breaking terms up to mass dimension of two to avoid the domain-wall problem. Since the scalar potential has a more general structure than that in the minimal model, the suppression of the DMnucleon scatterings in the low-energy limit is no longer guaranteed. We show that the scattering amplitudes mediated by the two scalar particles can still be cancelled when the masses of the two scalars are degenerate. In such a degenerate-scalar scenario we explore the parameter space which satisfies the direct detection constraints and the relic density. In addition, we discuss a possibility to verify such a scenario by using the recoil-mass technique at the International Linear Collider (ILC) [17]. We note that the most general model of pseudoscalar DM has recently been studied in Ref. [18] with a focus on the gravitationalwave signal. 
This paper is organized as follows. In Sec. II, we introduce our DM model. In Sec. III, we show the cancellation condition on the scattering between DM and quarks, and constraints on the parameter space from the direct detection experiments and the relic density of the DM. We discuss a possibility to verify a degenerate-scalar scenario at the ILC in Sec. IV. Section V is devoted to a summary.

\section{PSEUDOSCALAR DARK-MATTER MODEL}

We start with the following scalar potential of the CxSM [7],

$$
\begin{aligned}
V= & \frac{m^{2}}{2}|H|^{2}+\frac{\lambda}{4}|H|^{4}+\frac{\delta_{2}}{2}|H|^{2}|S|^{2}+\frac{b_{2}}{2}|S|^{2}+\frac{d_{2}}{4}|S|^{4} \\
& +\left(a_{1} S+\frac{b_{1}}{4} S^{2}+\text { c.c. }\right),
\end{aligned}
$$

where a global U(1) symmetry for the singlet $S$ is softly broken due to the linear $\left(a_{1}\right)$ and quadratic $\left(b_{1}\right)$ terms. ${ }^{1}$ All the coefficients in Eq. (1) are assumed to be real. We note that the minimal pseudo-Nambu-Goldstone DM model [6] forbids the linear term of $S$, i.e., $a_{1}=0$, where the scalar potential has a $Z_{2}$ symmetry $(S \rightarrow-S)$. This $Z_{2}$ symmetry is spontaneously broken by the VEV of the singlet $S$, and it causes the domain-wall problem [16]. The linear term $\left(a_{1} \neq 0\right)$ in the scalar potential, therefore, needs to break the $Z_{2}$ symmetry explicitly so that the domain-wall problem does not arise. Although it is worth considering the UV completion of the scalar potential (1), including the linear term of $S$, we do not discuss it further.

The SM-Higgs field $H$ and the singlet field $S$ are expressed in terms of the component fields as

$$
H=\frac{1}{\sqrt{2}}\left(\begin{array}{c}
0 \\
v+h
\end{array}\right), \quad S=\frac{1}{\sqrt{2}}\left(v_{S}+s+i \chi\right),
$$

where we adopt the unitary gauge so that the Goldstone fields in $H$ are suppressed. The VEVs of $H$ and $S$ are denoted by $v$ and $v_{S}$, respectively. The minimization conditions of the scalar potential $\left(\frac{\partial V}{\partial H}=0, \frac{\partial V}{\partial S}=0\right)$ leads to the following relations among parameters in Eq. (1),

$$
\begin{gathered}
-m^{2}=\frac{\lambda}{2} v^{2}+\frac{\delta_{2}}{2} v_{S}^{2}, \\
-b_{2}=\frac{\delta_{2}}{2} v^{2}+\frac{d_{2}}{2} v_{S}^{2}+b_{1}+2 \sqrt{2} \frac{a_{1}}{v_{S}} .
\end{gathered}
$$

\footnotetext{
${ }^{1}$ There are other renormalizable operators which break the global U(1) symmetry. But we have only adopted terms that close under renormalization [7]. The study of Ref. [18] includes all operators which we have dropped in the scalar potential Eq. (1).
}

The mass matrix $M^{2}$ of the charge and parity $(C P)$-even scalars $(h, s)$ is given by

$$
M^{2}=\left(\begin{array}{cc}
\frac{\lambda}{2} v^{2} & \frac{\delta_{2}}{2} v v_{S} \\
\frac{\delta_{2}}{2} v v_{S} & \Lambda^{2}
\end{array}\right),
$$

where $\Lambda^{2}$ is defined as

$$
\Lambda^{2} \equiv \frac{d_{2}}{2} v_{S}^{2}-\sqrt{2} \frac{a_{1}}{v_{S}} .
$$

The mass eigenstates $\left(h_{1}, h_{2}\right)$ are defined by using the orthogonal matrix $O$

$$
\left(\begin{array}{l}
h_{1} \\
h_{2}
\end{array}\right)=O\left(\begin{array}{l}
h \\
s
\end{array}\right), \quad O=\left(\begin{array}{cc}
\cos \alpha & \sin \alpha \\
-\sin \alpha & \cos \alpha
\end{array}\right) .
$$

Then, the mass eigenvalues $\left(m_{h_{1}}, m_{h_{2}}\right)$ and the mixing angle $\alpha$ are given by

$$
\begin{gathered}
m_{h_{1}, h_{2}}^{2}=\frac{1}{2}\left\{\frac{\lambda}{2} v^{2}+\Lambda^{2} \mp \sqrt{\left(\frac{\lambda}{2} v^{2}-\Lambda^{2}\right)^{2}+\left(\delta_{2} v v_{S}\right)^{2}}\right\}, \\
\cos 2 \alpha=\frac{\frac{\lambda}{2} v^{2}-\Lambda^{2}}{m_{h_{1}}^{2}-m_{h_{2}}^{2}} .
\end{gathered}
$$

Since the global U(1) symmetry is softly broken, the $C P$-odd scalar $\chi$ has a mass $m_{\chi}$ as

$$
m_{\chi}^{2}=-b_{1}-\sqrt{2} \frac{a_{1}}{v_{S}} .
$$

The $C P$ symmetry of the scalar potential (1) forbids the pseudoscalar $\chi$ to decay into the $C P$-even scalars so that $\chi$ could be identified as a DM particle.

We give the interaction Lagrangians which are necessary to our study below. The scalar-trilinear interactions for $\chi$ and $h_{1}$ or $h_{2}$ are given by

$$
\begin{aligned}
\mathcal{L}_{S}= & -\frac{1}{2 v_{S}}\left\{\left(m_{h_{1}}^{2}+\frac{\sqrt{2} a_{1}}{v_{S}}\right) \sin \alpha h_{1} \chi^{2}\right. \\
& \left.+\left(m_{h_{2}}^{2}+\frac{\sqrt{2} a_{1}}{v_{S}}\right) \cos \alpha h_{2} \chi^{2}\right\} .
\end{aligned}
$$

The Yukawa interactions of a fermion $f$ and the $C P$-even scalar $h_{1}$ or $h_{2}$ are given by

$$
\mathcal{L}_{Y}=-\frac{m_{f}}{v} \bar{f} f\left(h_{1} \cos \alpha-h_{2} \sin \alpha\right),
$$

where $m_{f}$ denotes the mass of the fermion $f$.

Here, we summarize the theoretical constraints on the model parameters. In the scalar potential, the quartic 
couplings $\lambda$ and $d_{2}$ are required to satisfy the following conditions from the perturbative unitarity [19]

$$
\lambda<\frac{16 \pi}{3}, \quad d_{2}<\frac{16 \pi}{3} .
$$

Also the stability condition of the scalar potential is given by [7]

$$
\lambda\left(d_{2}-\frac{2 \sqrt{2} a_{1}}{v_{S}^{3}}\right)>\delta_{2}^{2}
$$

We finally summarize the model parameters which will be used in the following analyses. There are seven parameters in the scalar potential and two VEVs. Since there are two minimization conditions on the scalar potential in Eqs. (3) and (4), the number of the model parameters is seven. We take $v \simeq 246 \mathrm{GeV}$ and identify $h_{1}$ as a scalar particle which has been discovered at the LHC with $m_{h_{1}} \simeq 125 \mathrm{GeV}$. We choose the remaining five parameters

$$
m_{h_{2}}, \quad m_{\chi}, \quad \alpha, \quad a_{1}, \quad v_{S},
$$

as inputs. so that other parameters

$$
m^{2}, \quad \lambda, \quad \delta_{2}, \quad b_{2}, \quad d_{2}, \quad b_{1},
$$

are given as functions of (15). It is convenient to express output parameters (16) by inputs (15) for later discussions;

$$
\begin{gathered}
m^{2}=-\frac{\lambda}{2} v^{2}-\frac{\delta_{2}}{2} v_{S}^{2}, \\
\lambda=\frac{2}{v^{2}}\left(m_{h_{1}}^{2} \cos ^{2} \alpha+m_{h_{2}}^{2} \sin ^{2} \alpha\right), \\
\delta_{2}=\frac{\frac{\lambda}{2} v^{2}-\Lambda^{2}}{v v_{S}} \tan 2 \alpha, \\
b_{2}=-\frac{\delta_{2}}{2} v^{2}-\frac{d_{2}}{2} v_{S}^{2}-b_{1}-2 \sqrt{2} \frac{a_{1}}{v_{S}}, \\
d_{2}\left(\frac{m_{h_{1}}^{2}}{v_{S}}\right)^{2} \sin ^{2} \alpha+2\left(\frac{m_{h_{2}}^{2}}{v_{S}}\right)^{2} \cos ^{2} \alpha+2 \sqrt{2} \frac{a_{1}}{v_{S}^{3}}, \\
b_{1}=-m_{\chi}^{2}-\sqrt{2} \frac{a_{1}}{v_{S}} .
\end{gathered}
$$

\section{SUPPRESSION OF DM-NUCLEON SCATTERING}

In this section, we discuss a suppression mechanism of the scattering process of the DM $\chi$ off a quark $q, \chi q \rightarrow \chi q$, whose Feynman diagram is shown in Fig. 1. We also discuss constraints on the model parameter space from

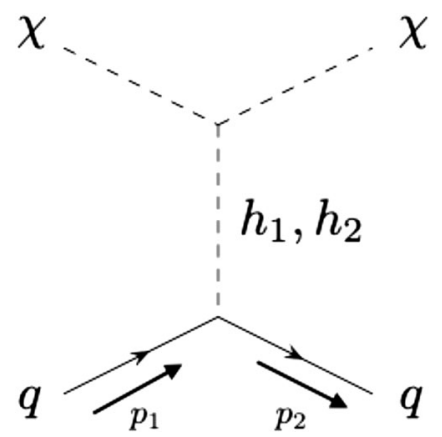

FIG. 1. Feynman diagram of the scattering process $\chi q \rightarrow \chi q$ mediated by $C P$-even scalars $h_{1}$ and $h_{2}$.

the direct detection experiments and the relic density of the DM.

The amplitudes mediated by $h_{1}$ and $h_{2}$ are given by

$i \mathcal{M}_{h_{1}}=-i \frac{m_{q}}{v v_{S}} \frac{m_{h_{1}}^{2}+\frac{\sqrt{2} a_{1}}{v_{S}}}{t-m_{h_{1}}^{2}} \sin \alpha \cos \alpha \bar{u}\left(p_{2}\right) u\left(p_{1}\right)$,

$i \mathcal{M}_{h_{2}}=+i \frac{m_{q}}{v v_{S}} \frac{m_{h_{2}}^{2}+\frac{\sqrt{2} a_{1}}{v_{S}}}{t-m_{h_{2}}^{2}} \sin \alpha \cos \alpha \bar{u}\left(p_{2}\right) u\left(p_{1}\right)$,

where $t \equiv\left(p_{1}-p_{2}\right)^{2}$, and $p_{1}\left(p_{2}\right)$ is the momentum of quark $q$ in the initial (final) state. Since the momentum transfer $t$ in the process is very small with respect to the mass of the scalars $m_{h_{1,2}}$, the sum of the two amplitudes can be written as

$$
\begin{aligned}
i\left(\mathcal{M}_{h_{1}}+\mathcal{M}_{h_{2}}\right) \simeq & i \frac{m_{q}}{v v_{S}} \sin \alpha \cos \alpha \bar{u}\left(p_{2}\right) u\left(p_{1}\right) \\
& \times \frac{1}{m_{h_{1}}^{2} m_{h_{2}}^{2}}\left\{\left(m_{h_{2}}^{2}-m_{h_{1}}^{2}+\frac{\sqrt{2} a_{1}}{v_{S}} \frac{m_{h_{2}}^{4}-m_{h_{1}}^{4}}{m_{h_{1}}^{2} m_{h_{2}}^{2}}\right) t\right. \\
& \left.+\frac{\sqrt{2} a_{1}}{v_{S}}\left(m_{h_{2}}^{2}-m_{h_{1}}^{2}\right)\right\} .
\end{aligned}
$$

The first term in the curly braces in the right-hand side is negligible when $t \simeq 0$ as shown in Ref. [6]. The second term, on the other hand, vanishes when the two scalars are degenerate $\left(m_{h_{1}}=m_{h_{2}}\right)$. The cancellation of the two amplitudes in the degenerate limit is by virtue of the orthogonal condition of the matrix $O$ in Eq. (7), $O_{i k} O_{j k}=\delta_{i j}$. Constraints on this model from the direct detection experiments, therefore, are weakened when the mass of the $C P$-even scalar $h_{2}$ is close to the mass of $h_{1}$, $m_{h_{1}} \simeq 125 \mathrm{GeV}$. This motivates us to study the phenomenological consequences of such a degenerate-scalar scenario in the pseudoscalar DM model. We note that in the $m_{h_{1}} \approx m_{h_{2}}$ case the two amplitudes in Eqs. (23) and (24) are cancelled not only for low energy but also for any energy 

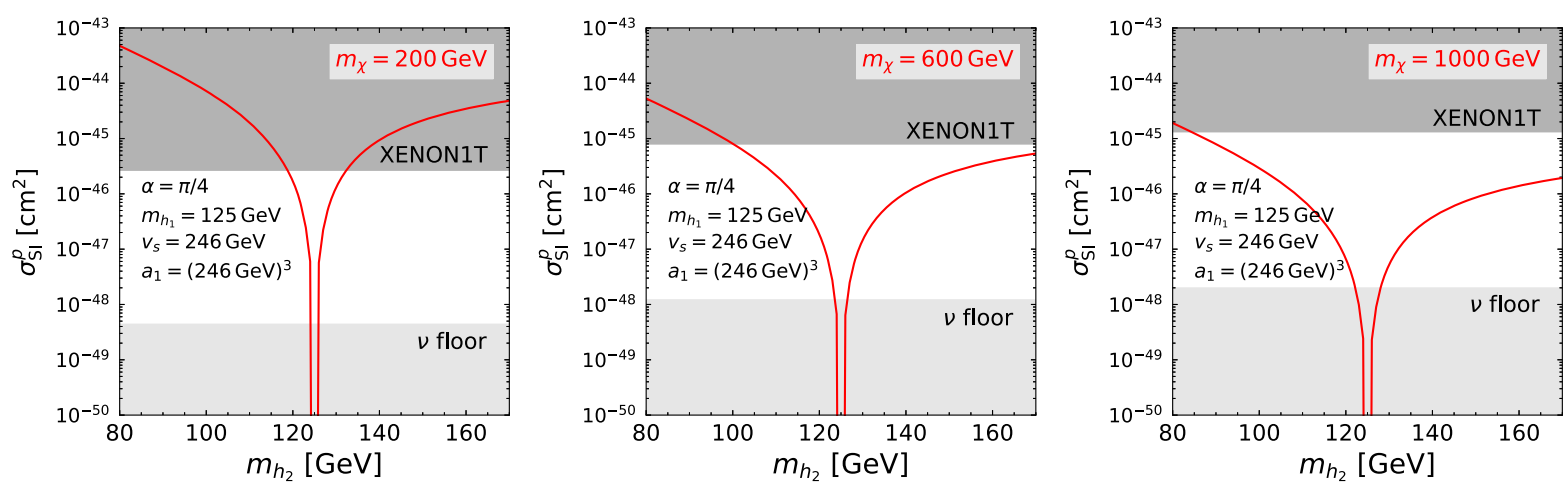

FIG. 2. Spin-independent DM-nucleon scattering cross section as a function of $m_{h_{2}}$ for the DM mass fixed at 200, 600, and $1000 \mathrm{GeV}$ from the left to right panels, respectively.

scale. This fact is relevant to understand the DM relic density in this model, which will be discussed later.

Before turning to numerical studies, we note that the scattering between DM and quarks at the one-loop level in the minimal model of [6] has been studied in Refs. $[9,10,15]$. Although the cancellation of the amplitudes at the low-energy limit does not hold any more at the one-loop level, the contributions are small enough to be neglected. For the $m_{h_{1}} \approx m_{h_{2}}$ case, especially, those contributions are strongly suppressed [9], which can be applied for our degenerate scenario even at the one-loop level.

In the following numerical studies, we implemented the pseudoscalar-DM model (i.e., the $\mathrm{CxSM}$ ) as the UFO format [20] by using FeynRules v2.3 [21], and used it in MADDM v3.0 [22-24] to compute the DM-nucleon scattering cross section and the relic density of DM.

In Fig. 2, we show the spin-independent cross section for the scattering between the DM and a nucleon $\sigma_{\mathrm{SI}}^{p}$ as a function of $m_{h_{2}}$. Three figures correspond to the DM mass $m_{\chi}=200 \mathrm{GeV}$ (left), $600 \mathrm{GeV}$ (center), and $1000 \mathrm{GeV}$ (right), respectively. Other parameters are fixed at $\alpha=\pi / 4$, $v_{S}=\sqrt[3]{a_{1}}=246 \mathrm{GeV}$ as an example. The upper and lower shaded regions represent the excluded region by the XENON1T experiment [2] and the background from the elastic neutrino-nucleus scattering (the so-called neutrino floor) [25]. As $a_{1} \neq 0$ in our model, the larger the mass difference between $h_{1}$ and $h_{2}$ is, the larger the DM-nucleon scattering cross section is. As expected, the cross section is highly suppressed around $m_{h_{2}} \sim m_{h_{1}}$.

We now examine dependences of the DM-nucleon cross section on the other parameters. In the following analyses we set the mixing angle $\alpha=\pi / 4$, where the cross section is maximal. Then, the remaining four parameters in (15); the mass difference $\Delta m=m_{h_{2}}-m_{h_{1}}$ (instead of $m_{h_{2}}$ ), the DM mass $m_{\chi}$, the tadpole coupling $a_{1}$, and the VEV of the singlet field $v_{S}$ are constrained from the experimental data.

In Fig. 3, we show contour plots of the spin-independent cross section $\sigma_{\mathrm{SI}}^{p}$ on $m_{\chi}-v_{S}$ (top), $m_{\chi}-\sqrt[3]{a_{1}}$ (middle), and $v_{s}-\sqrt[3]{a_{1}}$ (bottom) planes. The upper limit on $\sigma_{\mathrm{SI}}^{p}$ from XENON1T [2] is shown by the black-solid curve while the dotted-curve denotes the neutrino floor [25]. The mass difference $\Delta m$ is fixed at $1 \mathrm{GeV}$ for all the panels.

Three graphs in the first row of Fig. 3 correspond to $a_{1}=(123 \mathrm{GeV})^{3},(246 \mathrm{GeV})^{3}$, and $(492 \mathrm{GeV})^{3}$, respectively. In these graphs, the cross section $\sigma_{\mathrm{SI}}^{p}$ decreases for larger $v_{S}$. The reason can be understood as follows. For larger $v_{S}$, the $\chi-\chi-h_{i}$ interactions are relatively dominated by the $\chi-\chi-s$ interaction, because it is proportional to $v_{S}$ while the $\chi-\chi-h$ interaction is proportional to $v{ }^{2}$ Since the singlet $s$ does not interact with quarks, the scattering processes between the DM and quarks are suppressed for larger $v_{S}$. This is why the scattering amplitude of the DM and quarks in Eq. (25) is inversely proportional to $v_{S}$. On the other hand, the cross section increases as $a_{1}$ increases from the left to right panels. This is because the scattering amplitude of Eq. (25) is proportional to $a_{1}$. As seen, for the large $a_{1}$ case, the small $m_{\chi}$ and $v_{s}$ region is already excluded by the current direct detection experiment. Otherwise, the whole parameter space on this plane satisfies the constraint.

The second and third rows of Fig. 3 show the cross section $\sigma_{\mathrm{SI}}^{p}$ for $v_{S}=(123,246,492) \mathrm{GeV}$ and $m_{\chi}=$ $(200,600,1000) \mathrm{GeV}$, respectively. The common feature of these graphs is that the cross section increases as $a_{1}$ increases, as already mentioned above. The larger $m_{\chi}$ and $v_{s}$ are, the smaller the cross section becomes. We note that the theoretical constraints in Eqs. (13) and (14) are also satisfied on the entire parameter space presented in Fig. 3.

In Fig. 4 we show the $\Delta m$-dependence of the cross section $\sigma_{\mathrm{SI}}^{p}$ on the $m_{\chi}-v_{S}$ plane. We adopt cases in the first row of Fig. 3 as examples to discuss the $\Delta m$-dependence so that we list them on the first row of Fig. 4. Three different values of $\Delta m$ is chosen as $\Delta m=1 \mathrm{GeV}$ (top), $0.5 \mathrm{GeV}$ (middle), and $0.2 \mathrm{GeV}$ (bottom), and $a_{1}$ is $(123 \mathrm{GeV})^{3}$ (left), $(246 \mathrm{GeV})^{3}$ (center), and $(492 \mathrm{GeV})^{3}$ (right). While the degree of the degeneracy between the two scalars is

\footnotetext{
${ }^{2}$ Recall that $\chi-\chi-s$ and $\chi-\chi-h$ interactions are given by $|S|^{4}$ and $|H|^{2}|S|^{2}$ terms in (1), respectively.
} 

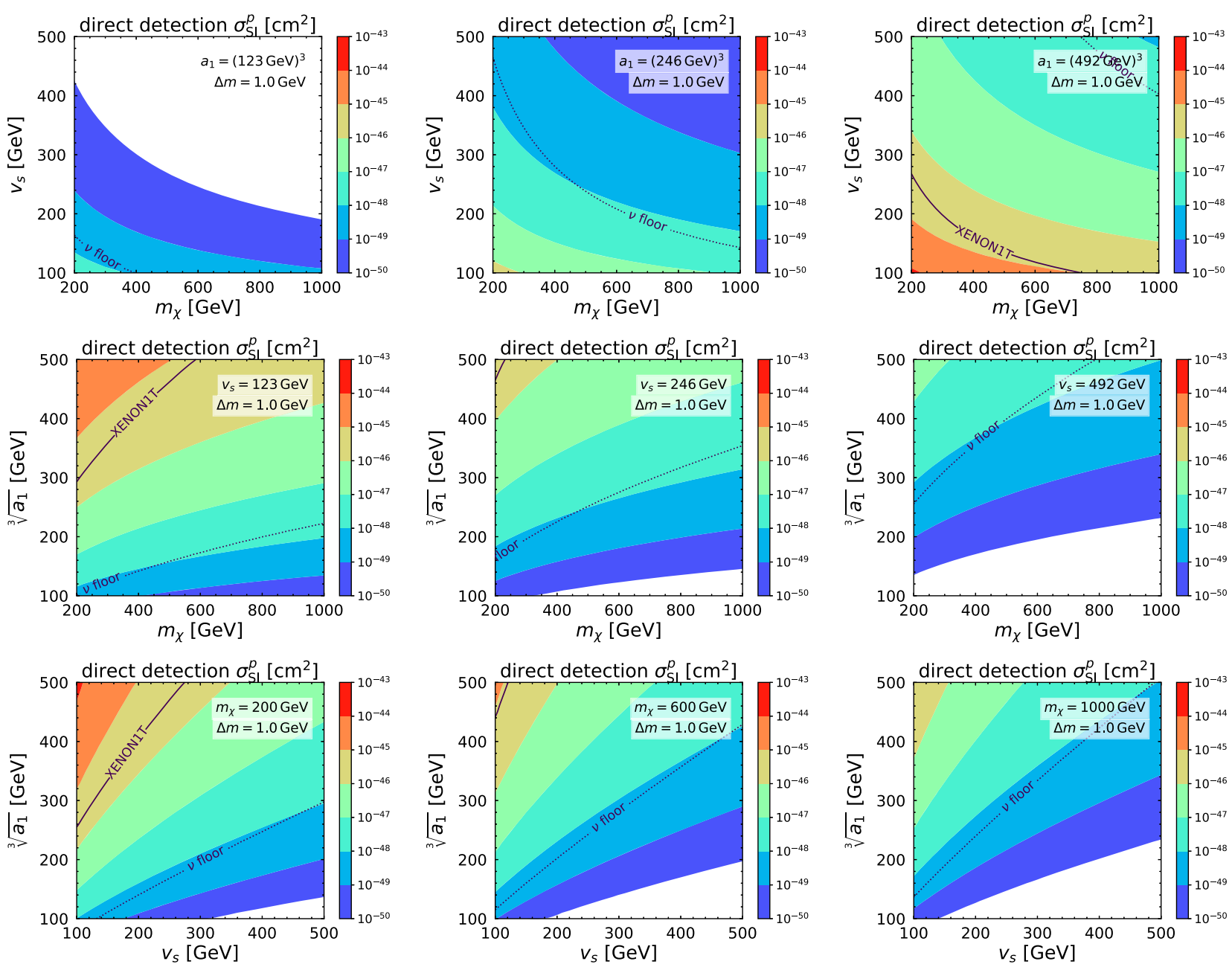

FIG. 3. Spin-independent DM-nucleon scattering cross section on the $m_{\chi}-v_{s}$ (top), $m_{\chi}-\sqrt[3]{a_{1}}$ (middle), and $v_{s}-\sqrt[3]{a_{1}}$ (bottom) planes. $\Delta m=m_{h_{2}}-m_{h_{1}}$ is fixed at $1 \mathrm{GeV}$ for all the panels, while the other parameter, $a_{1}$ (top), $v_{s}$ (middle), and $m_{\chi}$ (bottom), is taken as the low to high scale from the left to right panels.

increasing, the cross section drops sharply, as already seen in Fig. 2. For $\Delta m=0.2 \mathrm{GeV}$, the whole parameter space considered in Fig. 4 is no longer constrained, except a tiny corner of the small $m_{\chi}$ and $v_{S}$ for $a_{1}=(492 \mathrm{GeV})^{3}$.

Up to now, we have studied the parameter space of the pseudoscalar DM model with nearly degenerate scalars and found that sizable parameter regions are allowed from the direct detection experiments. In Fig. 5 we here show constraints on parameters $\left(m_{\chi}, v_{s}, a_{1}\right)$ from the relic density of the DM $\Omega_{\chi} h^{2}$. In the figure, contour plots of $\Omega_{\chi} h^{2}$ on $m_{\chi}-v_{S}, m_{\chi}-\sqrt[3]{a_{1}}$, and $v_{s}-\sqrt[3]{a_{1}}$ planes are shown in the first, second and third rows, respectively. The parameter choices are same as in Fig. 3. We note that, although we set $\Delta m=$ $1 \mathrm{GeV}$ as a benchmark, the results are not altered for $\Delta m=$ $0.5 \mathrm{GeV}$ or $0.2 \mathrm{GeV}$, different from the DM-nucleon scattering cross section as shown in Fig. 4. The black curve denotes $\Omega_{\chi} h^{2}=0.120$ from the Planck measurement [1], and the region of $\Omega_{\chi} h^{2}>0.120$ is disfavored since the DM is overproduced in this model.
From the first row in Fig. 5, we see that the relic density $\Omega_{\chi} h^{2}$ could be large as $v_{S}$ increases, while small as $a_{1}$ increases. This can be explained as follows. The dominant annihilation processes are $\chi \chi \rightarrow h_{i} h_{j}(i, j=1,2)$ since the processes for other final states $(q q, \ell \ell, V V)$ are strongly suppressed by the same mechanism with the case of the DM-quark scattering $\chi q \rightarrow \chi q$, where $q, \ell$, and $V$ stand for quarks, leptons and vector bosons, respectively. The scalar trilinear interactions for $h_{1}-\chi-\chi$ and $h_{2}-\chi-\chi$ in Eq. (11) are proportional to a ratio $a_{1} / v_{S}$. Therefore, larger $v_{S}$ suppresses the annihilation rate of the DM, while for larger $a_{1}$ the scalar trilinear interactions become stronger and the annihilation of the DM pair is enhanced. The similar behaviors can be observed in the second and third rows, i.e., on the different parameter planes.

One can see a strip in some particular parameter regions, where the relic density suddenly becomes large, e.g., for $m_{\chi}=300 \mathrm{GeV}-400 \mathrm{GeV}$ on the top-right panel in Fig. 5 . This can be explained by the suppression of the annihilation 

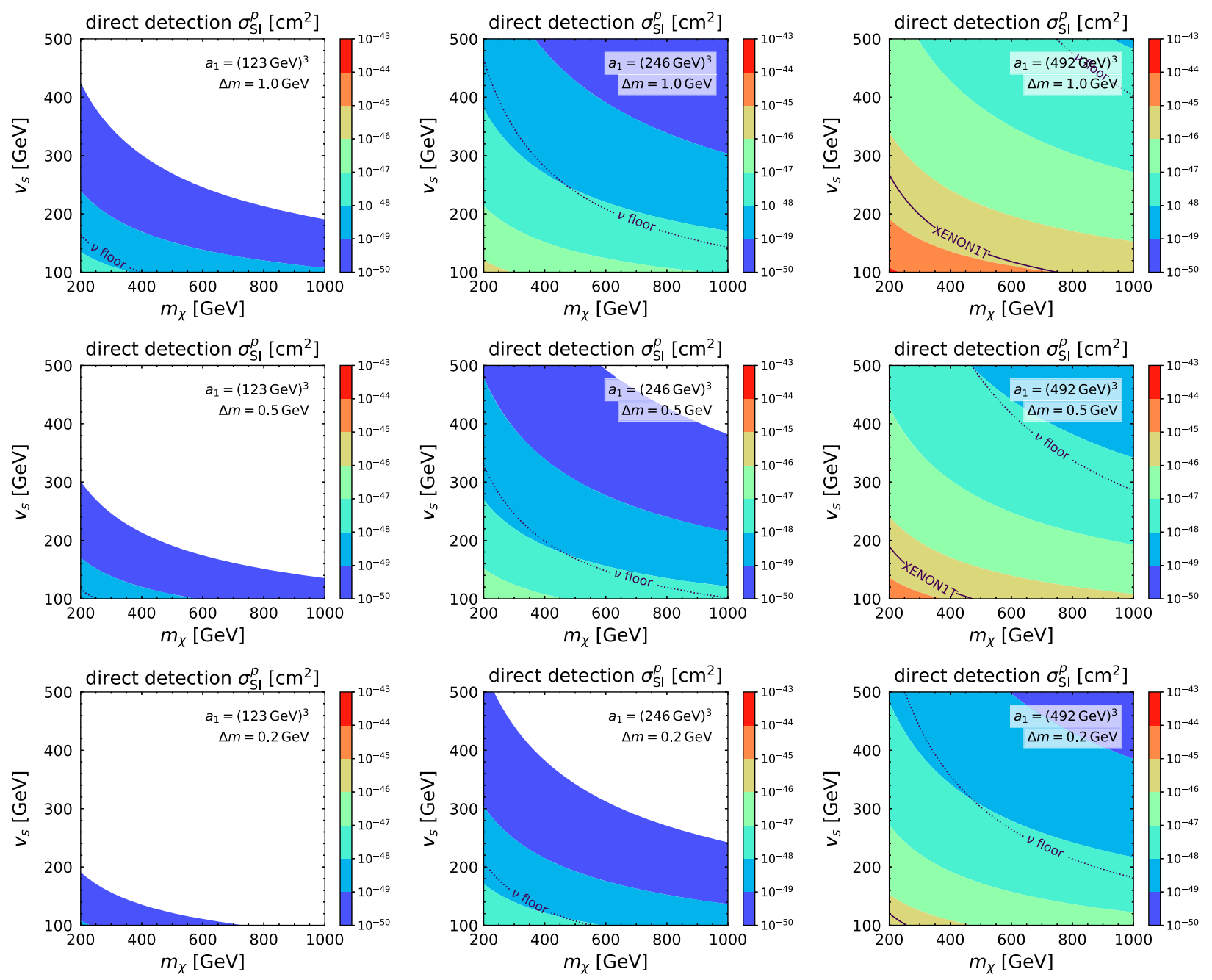

FIG. 4. Spin-independent DM-nucleon scattering cross section on the $m_{\chi}-v_{s}$ plane for $\Delta m=1.0,0.5,0.2 \mathrm{GeV}$ from the top to bottom panels, where $a_{1}$ is set as $(123 \mathrm{GeV})^{3},(246 \mathrm{GeV})^{3},(492 \mathrm{GeV})^{3}$ from the left to right panels.

cross section due to a nontrivial cancellation among the $\chi \chi \rightarrow h_{i} h_{j}$ amplitudes; the four-point, $s$-channel $h_{i}$-mediated, and $t$-channel $\chi$-mediated amplitudes.

In Fig. 5 we overlay the exclusion regions from the XENON1T direct detection experiment, shown in Fig. 3. The exclusion regions from the direct detections tend to correspond to the regions where the relic density is very small, and vice versa. The wide parameter regions of $\left(m_{\chi}, v_{s}, \sqrt[3]{a_{1}}\right)$ are still unconstrained from both the direct DM detection experiments and the measurement of the DM relic density. If a DM signal at direct detection experiments is observed in near future, the parameter space in this model can be narrowed down.

Before closing this section, we briefly mention a case for the low DM mass, especially for around $m_{\chi} \sim m_{h_{1}} / 2$. In Fig. 6 we show contour plots of the spin-independent cross section $\sigma_{\mathrm{SI}}^{p}$ (left) and the relic density of the DM $\Omega_{\chi} h^{2}$ (right) on $m_{\chi}-m_{h_{2}}$ plane for $v_{S}=\sqrt{3} a_{1}=246 \mathrm{GeV}$. As for the DM-nucleon scattering cross section, the suppression around $m_{h_{2}} \sim m_{h_{1}}$ can also be seen in this low $m_{\chi}$ region, while the $m_{\chi}$ dependence is very mild. On the other hand, the relic density strongly depends on $m_{\chi}$ as well as $m_{h_{2}}$. As seen, in this low $m_{\chi}$ case, the degenerate scalar scenario, $m_{h_{2}} \sim m_{h_{1}}$, is excluded by the limit from the relic density. This is because, different from the case shown in Fig. 5, the DM annihilation to the fermions and the gauge bosons are dominant, and are strongly suppressed in this scenario. Apart from the case of $m_{h_{2}} \sim m_{h_{1}}$, especially for $m_{\chi} \sim m_{h_{1}} / 2$ or $m_{\chi} \sim m_{h_{2}} / 2$, the relic density becomes very low since the DM annihilation is enhanced by the scalar resonance, which can be commonly observed in Higgsportal DM models [26].

\section{TEST FOR A DEGENERATE-SCALAR SCENARIO AT THE ILC}

We have so far discussed that the stringent constraints on DM models from direct detection experiments could be avoided in the pseudoscalar DM model with nearly degenerate scalars without contradicting the observed relic 

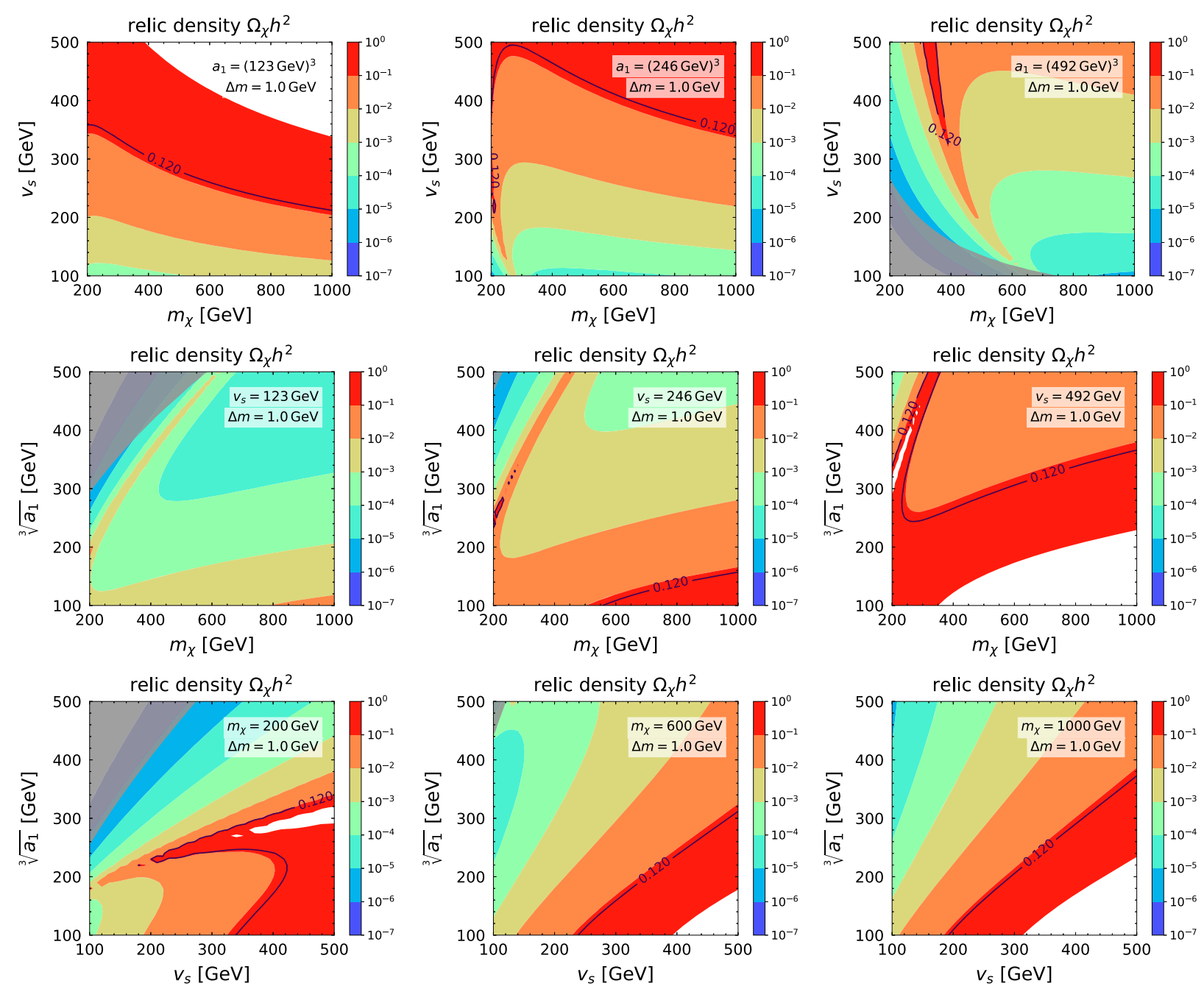

FIG. 5. DM relic density on the $m_{\chi}-v_{s}$ (top), $m_{\chi}-\sqrt[3]{a_{1}}$ (middle), and $v_{s}-\sqrt[3]{a_{1}}$ (bottom) planes. $\Delta m=m_{h_{2}}-m_{h_{1}}$ is fixed at $1 \mathrm{GeV}$ for all the panels, while the other parameter, $a_{1}$ (top), $v_{s}$ (middle), and $m_{\chi}$ (bottom), is taken as the low to high scale from the left to right panels. Gray shaded regions denote the region excluded by the XENON1T direct detection experiment.
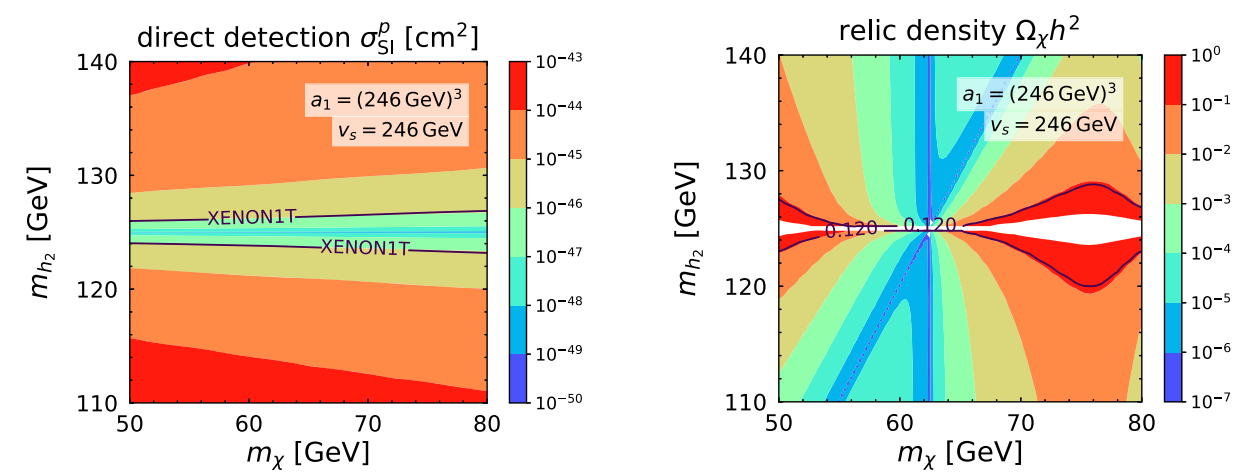

FIG. 6. Spin-independent DM-nucleon scattering cross section (left) and DM relic density (right) on the $m_{\chi}-m_{h_{2}}$ plane for the low $m_{\chi}$ region.

density of the DM as well as theoretical constraints on the scalar potential. In this section we discuss possibilities to verify such a degenerate-scalar scenario in collider experiments.
A typical DM signature at colliders is missing energy from DM production. In the pseudoscalar DM model, the DM $\chi$ (the $C P$-odd scalar) only couples to the $C P$-even scalars $h_{1}$ and $h_{2}$ (the SM-like $125 \mathrm{GeV}$ Higgs boson and 
the other scalar). Therefore, unless $m_{\chi}>m_{h_{1,2}} / 2$, the $h_{1,2}$ productions followed by their decays into a pair of $\chi$ can lead to such a missing-energy signature. The degeneratescalar case, especially, can be observed as invisible Higgs decay. We note that in such a case it is very difficult to distinguish the model from a simpler Higgs portal DM model, where DM only couples to the single SM-like Higgs boson. In the following, however, we will show that it is possible to examine the degenerate-scalar scenario using the process other than the invisible decay.

In order to test a degenerate-scalar scenario, instead of the missing-energy signature, here we focus on degeneratescalar productions at the ILC [27], and investigate how well we can distinguish the degenerate states from the single state of $m_{h}=125 \mathrm{GeV}$ in the SM. We note that, at the Large Hadron Collider (LHC), by using the high resolution of the diphoton channel of the Higgs boson decays, the mass difference between the two degenerate states $\Delta m \gtrsim 3 \mathrm{GeV}$ is disfavored at the $2 \sigma$ level from the LHC Run-I data [28]. Although no such specific analyses can be found in Refs. [29,30] with the LHC Run-II data, we expect a better resolution. The phenomenological studies on mass-degenerate Higgs bosons are also found in, e.g., Refs. [31-34].

The main target of the ILC at $\sqrt{s}=250 \mathrm{GeV}$ (ILC250) is Higgs boson production associated with a $Z$ boson [35]. The mass of the Higgs boson is precisely determined by the recoil-mass technique in the process [17]

$$
e^{+} e^{-} \rightarrow h_{1,2} Z \rightarrow h_{1,2} \ell^{+} \ell^{-},
$$

where the Higgs mass is reconstructed from $\ell=e$ or $\mu$ as

$$
m_{\text {recoil }}^{2}=\left(\sqrt{s}-E_{\ell \ell}\right)^{2}-\left|\vec{p}_{\ell \ell}\right|^{2} .
$$

The recoil mass is independent of how the degenerate scalars decay, and hence this analysis is independent of the mass hierarchy between the DM and the degenerate scalars. We note that, different from processes where the DM involves such as DM-nucleon scattering and DM annihilation, there is no cancellation between the two amplitudes mediated by $h_{1}$ and $h_{2}$ in the SM-like processes. ${ }^{3}$ In the following, we parametrize the mass difference of the two scalars as

$$
m_{h_{1}, h_{2}}=\left(125 \pm \frac{\Delta m}{2}\right) \mathrm{GeV}
$$

Figure 7 shows recoil-mass distributions with a muon-pair final state in the pseudoscalar DM model with nearly degenerate scalars for various $\Delta m$ denoted by different colors. For simulation, using the same CxSM

\footnotetext{
${ }^{3}$ Even when the $h_{1,2} \rightarrow \chi \chi$ decays are allowed in the process (26), unless the mass difference is smaller than the widths of $h_{1,2}$, no cancellation happens.
}

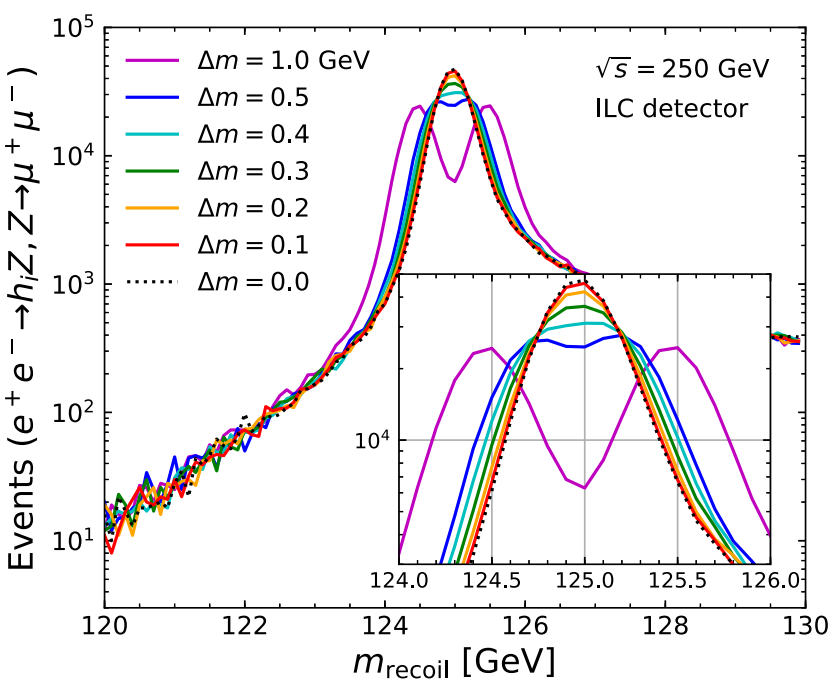

FIG. 7. Recoil-mass distributions for various $\Delta m$ for $e^{+} e^{-} \rightarrow$ $h_{1,2} Z, Z \rightarrow \mu^{+} \mu^{-}$at $\sqrt{s}=250 \mathrm{GeV}$, where the generic ILC detector is assumed.

UFO model as in the DM computations, we employed MadGraph5_aMC@NLO v2.7.3 [36] with PYTHIA 8.2 [37] for event generation and DELPHES v3.4.2 [38] to take into account detector effects. We used the ILCDELPHES card; a DELPHES model describing a parametrized generic ILC detector $[39,40]$ based on two types of detectors proposed for the ILC [41], the Silicon Detector (SiD) [42], and the International Large Detector (ILD) [43]. The total cross section for the signal, i.e., the sum of the two resonances is independent of $\Delta m$, while the relative strengths for the two resonances depend on the mixing angle $\alpha$. Here, as before, we assume $\alpha=\pi / 4$, i.e., the two resonances have the same signal strengths.

In Fig. 7, for $\Delta m=1.0 \mathrm{GeV}$, we can clearly observe two peaks. For $\Delta m<0.5 \mathrm{GeV}$, on the other hand, we no longer observe two peaks, instead see a broader peak than for the single resonance case denoted by a dotted curve $(\Delta m=0)$. We note that, as expected, the results strongly depend on the resolution of muon momenta determined by detectors. ${ }^{4}$

In order to assess the minimal luminosity to exclude or discover such a degenerate-scalar scenario, we perform tests of significance with the $\chi^{2}$ function defined as

$$
\chi^{2}=\sum_{i=1}^{N} \frac{\left(n^{i}-n_{\mathrm{SM}}^{i}\right)^{2}}{n_{\mathrm{SM}}^{i}}
$$

where $n^{i}$ is the number of events in the $i$ th bin expected in our pseudoscalar DM model, and $n_{\mathrm{SM}}^{i}$ is the corresponding

\footnotetext{
${ }^{4}$ In the early stage of this work, we performed two detector simulations, $\mathrm{SiD}$ and ILD, implemented as a DELPHES detector card in the previous ILC study [44]. The resolution of the recoilmass spectrum, i.e. the muon resolution, for SiD (ILD) is slightly worse (better) than that for the generic ILC detector.
} 

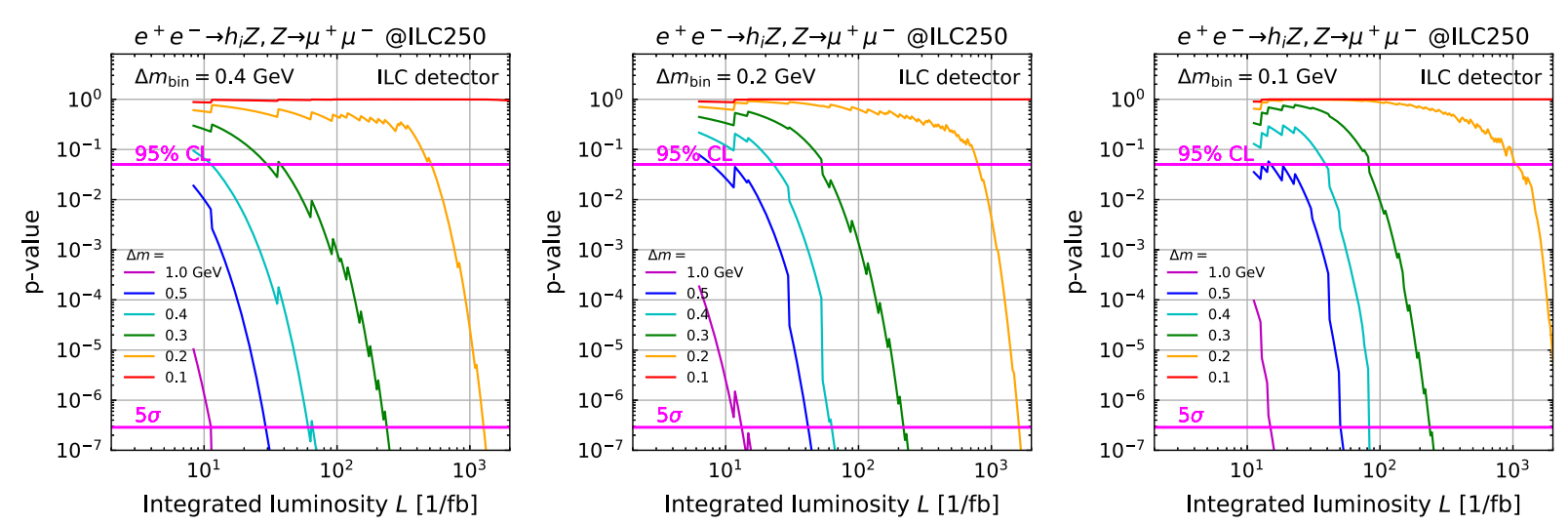

FIG. 8. The $p$-value for the distinction of the degenerate mass spectra as a function of the integrated luminosity for various $\Delta m$ with the generic ILC detector. From the left to right panels, the size of bins in the $120-130 \mathrm{GeV}$ range is taken as 0.4, 0.2, and $0.1 \mathrm{GeV}$, respectively.

prediction in the SM. As the background in the recoil-mass distribution is estimated well in ref. [17], we simply take the SM Higgs signal as our zero hypothesis. We fix the total number of events at a certain integrated luminosity by the total cross section $10 \mathrm{fb}\left[=\sigma\left(e^{+} e^{-} \rightarrow h Z\right) \times B\left(Z \rightarrow \mu^{+} \mu^{-}\right)\right]$ for $m_{h}=125 \mathrm{GeV}$ at $\sqrt{s}=250 \mathrm{GeV}$ with the planned beam polarization $P\left(e^{-}, e^{+}\right)=(-0.8,0.3)$ [35]. We require at least ten events in each bin to count as the degree of freedom for the $\chi^{2}$ calculation in Eq. (29).

Figure 8 (left) shows the $p$-value calculated from the $\chi^{2}$ for the distinction of the degenerate mass spectra of various $\Delta m$ as a function of the integrated luminosity $L$ up to $2 \mathrm{ab}^{-1}$ planned at the ILC250, where we take $N=25$ bins in the $120 \mathrm{GeV}-130 \mathrm{GeV}$ range, i.e., $\Delta m_{\text {bin }}=0.4 \mathrm{GeV}$. We find that, e.g., the scenario with $\Delta m<0.3 \mathrm{GeV}$ is excluded (discovered) with $L \sim 30 \mathrm{fb}^{-1}\left(240 \mathrm{fb}^{-1}\right)$, while that with $\Delta m<0.2 \mathrm{GeV}$ is $L \sim 520 \mathrm{fb}^{-1}\left(1300 \mathrm{fb}^{-1}\right)$.

We also investigate the effect of the bin size on the distinction; from the left to right panels, we take the number of bins in the $120 \mathrm{GeV}-130 \mathrm{GeV}$ range as $N=25,50$ and 100 , i.e. $\Delta m_{\text {bin }}=0.4,0.2,0.1 \mathrm{GeV}$, respectively. Even for $\Delta m_{\text {bin }}>\Delta m$, we expect a sensitivity to exclude or discover the degenerate-scalar scenario within the planned integrated luminosity $2 \mathrm{ab}^{-1}$ if $\Delta m>0.2 \mathrm{GeV}$. A better sensitivity can be expected with more optimized analyses, and more dedicated studies such as including the background and the systematic errors should be reported elsewhere.

\section{SUMMARY}

In this paper, we studied a pseudoscalar DM model arising from the CxSM. The scalar potential we adopted in our study is the most general and renormalizable, in which the global symmetry is softly broken by the operators up to the mass dimension two so that does not suffer from the domain-wall problem as opposed to the minimal pseudo-Nambu-Goldstone DM model. We showed that the DM-nucleon scattering amplitudes mediated by two scalar particles $\left(h_{1}\right.$ and $\left.h_{2}\right)$ are cancelled when the masses of two scalars are degenerate, and we investigated the allowed model parameter space of the degenerate scenario under the direct-detection experiments as well as the measurements of the relic density of the DM.

In addition, we discussed a possibility to verify such a degenerate-scalar scenario by using the recoil-mass technique at the ILC. The recoil mass is independent of how the degenerate scalars decay, and hence the analysis is independent of the mass hierarchy between the DM and the degenerate scalars in the model. We found that a pair of states separated by $0.2 \mathrm{GeV}$ can be distinguished from the single SM-like Higgs state at $5 \sigma$ with integrated luminosity of $2 \mathrm{ab}^{-1}$.

Finally, we briefly mention constraints on the degenerate scalar scenario from so-called indirect detection experiments, which restrict the annihilation processes of the DM. We expect that the degenerate scalars suppress the annihilation rates of the DM pair to fermions or gauge bosons owing to the cancellation mechanism so that these processes are not severely constrained from the indirect detection experiments. This consequence is different from a typical Higgs portal DM model (e.g., [45]), which does not have such a cancellation mechanism. In the degenerate scenario, as is mentioned in Sec. III, the DM annihilation to scalar pairs, $\chi \chi \rightarrow h_{i} h_{j}$, is not suppressed. Therefore, the indirect detection experiments might give some constraints on the model parameter space; this will be reported elsewhere.

\section{ACKNOWLEDGMENTS}

We would like to thank Federico Ambrogi, Chiara Arina, Jan Heisig and Olivier Mattelaer for their valuable help with MADDM and Takanori Kono for many helpful discussions. We also thank Keisuke Fujii for useful information on the ILC. The work of G. C. C. is supported in part by Grants-in-Aid for Scientific Research from the Japan Society for the Promotion of Science (No. 16K05314). The work of K. M. is supported in part by JSPS KAKENHI Grants No. 18K03648, No. 20H05239, and No. 21H01077. 
[1] N. Aghanim et al. (Planck Collaboration), Planck 2018 results. VI. Cosmological parameters, Astron. Astrophys. 641, A6 (2020).

[2] E. Aprile et al. ( XENON Collaboration), Dark Matter Search Results from a One Ton-Year Exposure of XENON1T, Phys. Rev. Lett. 121, 111302 (2018).

[3] S. Ipek, D. McKeen, and A. E. Nelson, A renormalizable model for the galactic center gamma ray excess from dark matter annihilation, Phys. Rev. D 90, 055021 (2014).

[4] M. Escudero, A. Berlin, D. Hooper, and M.-X. Lin, Toward (finally!) ruling out $\mathrm{z}$ and higgs mediated dark matter models, J. Cosmol. Astropart. Phys. 12 (2016) 029.

[5] T. Abe, M. Fujiwara, and J. Hisano, Loop corrections to dark matter direct detection in a pseudoscalar mediator dark matter model, J. High Energy Phys. 02 (2019) 028.

[6] C. Gross, O. Lebedev, and T. Toma, Cancellation Mechanism for Dark-Matter-Nucleon Interaction, Phys. Rev. Lett. 119, 191801 (2017).

[7] V. Barger, P. Langacker, M. McCaskey, M. Ramsey-Musolf, and G. Shaughnessy, Complex Singlet Extension of the Standard Model, Phys. Rev. D 79, 015018 (2009).

[8] D. Azevedo, M. Duch, B. Grzadkowski, D. Huang, M. Iglicki, and R. Santos, Testing scalar versus vector dark matter, Phys. Rev. D 99, 015017 (2019).

[9] D. Azevedo, M. Duch, B. Grzadkowski, D. Huang, M. Iglicki, and R. Santos, One-loop contribution to darkmatter-nucleon scattering in the pseudo-scalar dark matter model, J. High Energy Phys. 01 (2019) 138.

[10] K. Ishiwata and T. Toma, Probing pseudo NambuGoldstone boson dark matter at loop level, J. High Energy Phys. 12 (2018) 089.

[11] K. Huitu, N. Koivunen, O. Lebedev, S. Mondal, and T. Toma, Probing pseudo-Goldstone dark matter at the LHC, Phys. Rev. D 100, 015009 (2019).

[12] T. Alanne, M. Heikinheimo, V. Keus, N. Koivunen, and K. Tuominen, Direct and indirect probes of Goldstone dark matter, Phys. Rev. D 99, 075028 (2019).

[13] J. M. Cline and T. Toma, Pseudo-Goldstone dark matter confronts cosmic ray and collider anomalies, Phys. Rev. D 100, 035023 (2019).

[14] C. Arina, A. Beniwal, C. Degrande, J. Heisig, and A. Scaffidi, Global fit of pseudo-Nambu-Goldstone dark matter, J. High Energy Phys. 04 (2020) 015.

[15] S. Glaus, M. Mühlleitner, J. Müller, S. Patel, T. Römer, and R. Santos, Electroweak Corrections in a Pseudo-Nambu Goldstone dark matter model revisited, J. High Energy Phys. 12 (2020) 034.

[16] Y. Zeldovich, I. Kobzarev, and L. Okun, Cosmological consequences of the spontaneous breakdown of discrete symmetry, Zh. Eksp. Teor. Fiz. 67, 3 (1974).

[17] J. Yan, S. Watanuki, K. Fujii, A. Ishikawa, D. Jeans, J. Strube, J. Tian, and H. Yamamoto, Measurement of the Higgs boson mass and $e^{+} e^{-} \rightarrow Z H$ cross section using $Z \rightarrow \mu^{+} \mu^{-}$and $Z \rightarrow e^{+} e^{-}$at the ILC, Phys. Rev. D 94, 113002 (2016).

[18] T. Alanne, N. Benincasa, M. Heikinheimo, K. Kannike, V. Keus, N. Koivunen, and K. Tuominen, Pseudo-Goldstone dark matter: Gravitational waves and direct-detection blind spots, J. High Energy Phys. 10 (2020) 080.
[19] C.-Y. Chen, S. Dawson, and I. Lewis, Exploring resonant diHiggs boson production in the Higgs singlet model, Phys. Rev. D 91, 035015 (2015).

[20] C. Degrande, C. Duhr, B. Fuks, D. Grellscheid, O. Mattelaer, and T. Reiter, UFO-The Universal FeynRules Output, Comput. Phys. Commun. 183, 1201 (2012).

[21] A. Alloul, N. D. Christensen, C. Degrande, C. Duhr, and B. Fuks, FeynRules 2.0-A complete toolbox for tree-level phenomenology, Comput. Phys. Commun. 185, 2250 (2014).

[22] M. Backovic, K. Kong, and M. McCaskey, MadDM v.1.0: Computation of dark matter relic abundance using MadGraph5, Phys. Dark Universe 5-6, 18 (2014).

[23] M. Backović, A. Martini, O. Mattelaer, K. Kong, and G. Mohlabeng, Direct detection of dark matter with MadDM v.2.0, Phys. Dark Universe 9-10, 37 (2015).

[24] F. Ambrogi, C. Arina, M. Backovic, J. Heisig, F. Maltoni, L. Mantani, O. Mattelaer, and G. Mohlabeng, MadDM v.3.0: A comprehensive tool for dark matter studies, Phys. Dark Universe 24, 100249 (2019).

[25] J. Billard, L. Strigari, and E. Figueroa-Feliciano, Implication of neutrino backgrounds on the reach of next generation dark matter direct detection experiments, Phys. Rev. D 89, 023524 (2014).

[26] G. Arcadi, A. Djouadi, and M. Raidal, Dark Matter through the Higgs portal, Phys. Rep. 842, 1 (2020).

[27] The International Linear Collider Technical Design Report-Volume 1: Executive summary, arXiv:1306.6327.

[28] V. Khachatryan et al. ( CMS Collaboration), Observation of the diphoton decay of the Higgs boson and measurement of its properties, Eur. Phys. J. C 74, 3076 (2014).

[29] M. Aaboud et al. ( ATLAS Collaboration), Measurement of the Higgs boson mass in the $H \rightarrow Z Z^{*} \rightarrow 4 \ell$ and $H \rightarrow \gamma \gamma$ channels with $\sqrt{s}=13 \mathrm{TeV} p p$ collisions using the ATLAS detector, Phys. Lett. B 784, 345 (2018).

[30] A. M. Sirunyan et al. ( CMS Collaboration), A measurement of the Higgs boson mass in the diphoton decay channel, Phys. Lett. B 805, 135425 (2020).

[31] J. F. Gunion, Y. Jiang, and S. Kraml, Diagnosing Degenerate Higgs Bosons at $125 \mathrm{GeV}$, Phys. Rev. Lett. 110, 051801 (2013).

[32] P. Ferreira, R. Santos, H. E. Haber, and J. P. Silva, Massdegenerate Higgs bosons at $125 \mathrm{GeV}$ in the two-Higgsdoublet model, Phys. Rev. D 87, 055009 (2013).

[33] T. Robens and T. Stefaniak, Status of the Higgs singlet extension of the Standard Model after LHC Run 1, Eur. Phys. J. C 75, 104 (2015).

[34] L. Bian, N. Chen, W. Su, Y. Wu, and Y. Zhang, Future prospects of mass-degenerate Higgs bosons in the $C P$-conserving two-Higgs-doublet model, Phys. Rev. D 97, 115007 (2018).

[35] K. Fujii et al., Physics case for the $250 \mathrm{GeV}$ stage of the international linear collider, arXiv:1710.07621.

[36] J. Alwall, R. Frederix, S. Frixione, V. Hirschi, F. Maltoni, O. Mattelaer, H.-S. Shao, T. Stelzer, P. Torrielli, and M. Zaro, The automated computation of tree-level and next-toleading order differential cross sections, and their matching to parton shower simulations, J. High Energy Phys. 07 (2014) 079. 
[37] T. Sjstrand, S. Ask, J. R. Christiansen, R. Corke, N. Desai, P. Ilten, S. Mrenna, S. Prestel, C. O. Rasmussen, and P. Z. Skands, An introduction to PYTHIA 8.2, Comput. Phys. Commun. 191, 159 (2015).

[38] J. de Favereau, C. Delaere, P. Demin, A. Giammanco, V. Lematre, A. Mertens, and M. Selvaggi (DELPHES 3 Collaboration), DELPHES 3, A modular framework for fast simulation of a generic collider experiment, J. High Energy Phys. 02 (2014) 057.

[39] K. Fujii et al., ILC study questions for snowmass 2021, arXiv:2007.03650.

[40] ILCDelphes: Delphes model describing a parametrised generic ILC detector; http://ilcsnowmass.org.
[41] H. Abramowicz et al., The International Linear Collider Technical Design Report-Volume 4: Detectors, arXiv: 1306.6329.

[42] H. Aihara et al., SiD letter of intent, arXiv:0911.0006.

[43] T. Abe et al. (Linear Collider ILD Concept GroupCollaboration), The International Large Detector: Letter of intent, arXiv:1006.3396.

[44] C. Potter, DSiD: A Delphes detector for ILC physics studies, in International Workshop on Future Linear Colliders, 2016, arXiv:1602.07748.

[45] M. Duerr, P. Fileviez Pérez, and J. Smirnov, Scalar dark matter: Direct vs indirect detection, J. High Energy Phys. 06 (2016) 152. 